\title{
Les noms du fond, ou la fonction des noms dits « sommitaux »
}

\author{
Marco Fasciolo ${ }^{1,1}$ \\ ${ }^{1}$ Sorbonne Université
}

Résumé. Cet article s'interroge sur la fonction des notions désignées par les noms généraux. Ces notions n'identifient pas des véritables concepts, mais bien des limites ontologiques à l'intérieur desquelles chaque lexique peut découper ses propres concepts grâce à des matrices de prédicats appropriés. Nulle surprise, donc, que les noms exprimant ces limites mal s'adaptent à classifier et manifestent un comportement pseudo pronominal.

\begin{abstract}
Ground nouns. This paper inquires into the function of general nouns' contents. These contents do not work as concepts capable of subsuming instances, but rather as ontological limits inside of which each lexicon can carve its own concepts out trough a matrix of appropriate predicates. No surprise, then, if the nouns expressing such limits look unfit to classification and show a pseudo-pronominal behaviour.
\end{abstract}

\section{Introduction}

Considérons les listes suivantes :

être humain, être vivant, objet concret, lieu...

tête de bétail, insecte, meuble, instrument de musique, agglomération...

vache, mouche, guitare, canapé, ville...

Face aux listes sous (0), on peut tracer deux ordres de distinctions. D'une part, on peut séparer les expressions (0b) de (0c) : dans la terminologie de Rosch (1976), les unes identifient des « superordinate categories», alors que les autres identifient des «basic level objects » (cf. également Kleiber 1994). D'autre part, on peut séparer (0a) de (0b) : les premières paraissent identifier des notions - si possible - encore plus superordonnées que les secondes : des notions tout à fait «sommitales». Cette contribution concerne ces dernières.

1 marco.fasciolo@gmail.com 
Les notions sommitales ont fait l'objet d'études dans différents cadres théoriques : pensons, par exemple, à Greenberg (1972), Harris (1976), Lyons (1977), IvaniČ (1991), Aikhenvald (2000) et Mahlberg (2005) entre autres. Même la grammaire générative des origines et le lexique génératif de Pustejovsky (1995), dans les faits, ont reconnu l'importance de ces notions générales en les incorporant implicitement dans les restrictions de sélection et dans les Qualia. Quoi qu'il en soit, à notre avis, l'auteur qui a mis en évidence les sommitaux de la façon la plus claire est Gross (2008, 2012). Cet auteur oppose explicitement des «hyper-classes », telles que $<$ humain $>$, < animal $>$, < végétal $>$, < <oncret $>$, <locatif $>$ et $<$ temps $>$, à des classes d'objets comme $<$ arbres $>$, $<$ herbes $>$, $<$ moyens de transport $>$, $<$ moyens de transport publics $>$, $<$ moyens de transport privés $>$, $<$ voies de communication $>$, $<$ boissons $>$, $<$ vêtements $>$, etc. Les premières sont bien des sommitaux.

La littérature spécialisée a remarqué à plusieurs reprises le statut borderline des noms exprimant les notions sommitales: leur semi-lexicalité ou semi-grammaticalité (Mihatsch 2007, 2015a,b, 2017), leur utilité pour la cohésion textuelle (Halliday\&Hasan 1960, Mahlberg 2005), leur comportement pseudo-pronominal (Gross 2009, Fasciolo 2012, Mihatsch 2017) et leur incompatibilité avec l'approximation (Fasciolo 2012, Gerhard-Krait \& Vassiliadou 2017). La contribution de ces travaux peut être définie " négative » dans la mesure où ils soulignent tous les différences du comportement des noms sommitaux par rapport aux autres. Notre contribution, en revanche, se voudrait "positive" - et complémentaire à la précédente - car nous nous proposons d'émettre une hypothèse concernant la fonction des notions désignées par les noms sommitaux, et d'interpréter les différences susmentionnées comme des conséquences de cette fonction.

La structure de cette contribution est la suivante. Tout d'abord $(\S 1)$, nous rappelons la fonction prototypique des noms : à savoir, classifier et non référer. Ensuite (\$2), nous séparons les noms sommitaux des autres sur la base du fait que les catégories désignées par les premiers ne remplissent pas bien la fonction de classifier. Sous $\S 3$, nous suggérons que ces catégories remplissent une autre fonction: déterminer des matrices de relations qui fixent les bornes de toute classification possible. Finalement ( $\$ 4)$, nous indiquons quelques pistes de recherche cohérentes avec notre hypothèse.

\section{Une fonction pour les noms : classifier}

Affirmer que les noms manifestent une affinité élective avec la fonction de référer peut paraître un truisme. Une telle affirmation, cependant, a deux inconvénients. Tout d'abord, elle néglige le fait que, pour pouvoir être employés dans un acte de référence, les noms (communs) ont besoin d'un déterminant. Ensuite, et surtout, elle cache la véritable fonction des noms : classifier. Considérons un groupe nominal comme (1) :

\section{Une étoile.}

En (1), il y a un partage du travail entre le déterminant et le nom : le déterminant une confère au nom étoile l'aptitude à être employé pour référer; le nom étoile offre un concept capable de classifier un référent. Si (1) est utilisé pour se référer au soleil (qui est un corps céleste et une étoile), la classification est cohérente et vraie. Si (1) est utilisé pour se référer à la lune (qui est un corps céleste et un satellite), la classification est cohérente et fausse. Si (1) est utilisé pour se référer à une fille (qui n'est pas un corps céleste), la classification est incohérente: le présupposé pour la vérité ou fausseté de toute classification est brisé. 
Un groupe nominal remplit alors non une, mais deux fonctions - référer et classifier assurées par des éléments différents: déterminant et nom. La fonction spécifique du nom n'est donc pas de référer, mais bien de classifieri.

La seule partie du discours dont la fonction spécifique est celle de référer n'est ni le nom, ni le déterminant, mais le pronom. A la différence des noms et des déterminants, les pronoms sont des expressions saturées, capables de remplir une fonction référentielle immédiatement ${ }^{\mathrm{ii}}$. Les pronoms, cependant, payent ce privilège en renonçant à la fonction de classifier. Transformer un groupe nominal en pronom revient en effet à supprimer précisement sa capacité de classifier :

$$
\begin{aligned}
& \text { Cette étoile brille } \rightarrow \underline{\text { Cette-ci brille. }} \\
& \text { Venus est } \underline{\text { une étoile }} \rightarrow \text { Venus est } \underline{\text { cette-ci. }} .
\end{aligned}
$$

Nous en retiendrons trois conclusions : $i$ ) la fonction prototypique des noms est de classifier; ii) la fonction prototypique des pronoms est de référer; iii) ces fonctions sont logiquement indépendantes, voire incompatibles sur une même unité lexicale. Une telle indépendance, par ailleurs, est la précondition pour pouvoir travailler en synergie dans une expression nominale telle que (1).

Dans le cadre à peine esquissé, le problème posé par noms sommitaux est que - tout en étant des noms communs et non des noms propres - ils sont récalcitrants à classifier : c'està-dire, à fonctionner comme des concepts capables de subsumer des occurrences.

\section{Des noms qui ne classifient pas}

Le $\S 2$ est consacré à illustrer la résistance que les noms sommitaux opposent à la classification. Sous \$2.1, nous avançons deux arguments visant à suggérer que leurs contenus ne fonctionnent pas comme des classes (ou concepts). Sous $\$ 2.2$, nous présentons deux phénomènes, concernant les usages de ces noms, cohérents avec l'hypothèse précédente.

\subsection{Du côté des contenus}

\subsubsection{Argument 'psychologique'}

Considérons les listes suivantes :

Guitare, harpe, trompette, piano, violon.

Paris, Rome, Londres, Amsterdam, Washington.

Soldat, amiral, sergent, colonel.

Intuitivement, les listes sous (4) seraient perçues comme étant sémantiquement homogènes. Les noms sous (4) seraient immédiatement catégorisés comme instruments de musique, villes et professions militaires. Ces expressions ne désignent évidemment pas des sommitaux au sens de notre introduction. Dans les termes de Gross (2008), il ne s'agit pas de « hyper-classes », mais bien de « classes d'objets ». 
Observons maintenant ces autres listes :

Guitare, arbre, table, pierre, bouteille.

Paris, Sahara, Champs-Élysées, Pole Nord.

Soldat, étudiant, avocat, voleur, plombier.

Par rapport à (4), les listes (5) paraissent plus hétérogènes. Remarquons qu'en principe rien n'empêche d'affirmer que leurs éléments sont tous des objets concrets, des lieux ou des humains. Cependant, on le fera seulement comme dernier recours, car on n'arrive pas à les classifier au niveau plus bas pertinent pour (4). Or, objets concrets, lieux et humains sont précisément des sommitaux : des « hyper-classes » dans la terminologie susmentionnée.

La différente perception des listes (4) et (5) suggère que nous cherchons à regrouper les entités au niveau des "classes d'objets » en nous appuyant sur les "hyper-classes ». Ce sont les premières - et non les secondes - qui sont immédiatement saillantes pour classifier.

\subsubsection{Argument 'cognitif'}

Considérons les hedges (Lakoff 1972) ou enclosures (Kleiber\&Martin 1978) suivants :

$$
\begin{aligned}
& \mathrm{x} \text { est un vrai } \mathrm{N} \\
& \mathrm{x} \text { est une sorte de } \mathrm{N}
\end{aligned}
$$

La structure (6a) communique que $x$ est particulièrement représentatif du concept contenu dans $N$. La structure (6b) - dans sa lecture d'approximation - communique que $x$ ressemble à un $N$, sans l'être tout à fait ${ }^{\text {iii }}$. Les deux structures s'appuient sur le fait que $N$ contient un concept, une classe. Or, si les sommitaux ne fonctionnent pas comme des véritables concepts, ils doivent manifester une certaine résistance à remplir cet $N$.

De manière générale, la prévision est confirmée ${ }^{\text {iv }}$ :

La Fender Stratocaster est une vraie guitare.

?La Fender Stratocaster est un vrai objet concret.

L'Opéra est un vrai théâtre.

?L'Opéra est un vrai lieu.

Une mandoline est une sorte de guitare, mais...

... est une sorte d'objet matériel, mais...

Un cinéma est une sorte de théâtre, mais...

... est une sorte de lieu, mais...

Comparées aux énoncés (a), les variantes (b) paraissent conceptuellement moins claires. Ainsi, par exemple, une mandoline peut être une réponse raisonnable à une question comme : qu'est-ce qui ressemble à une guitare sans l'être tout à fait? Mais il paraît plus 
problématique de répondre à : qu'est-ce qui ressemble à un lieu / à un objet matériel sans l'être tout à fait?

\subsection{Du côté des emplois}

Si les contenus des noms sommitaux ne sont pas saillants en tant que classes ou concepts, il est raisonnable de s'attendre à : $i$ ) ce qu'ils ne soient pas employés pour dénommer des classes ou des concepts (sous \$2.2.1) ; et : ii) ce qu'ils manifestent certains comportements typiques d'une partie du discours incompatible avec la classification, à savoir les pronoms (sous $§ 2.2 .2$ ).

\subsubsection{Dénomination}

En nous basant sur les tests de dénomination proposés par Kleiber (1987), observons les exemples suivants :

Comment les guitares s'appellent-elles en italien?

?Comment les objets s'appellent-ils en italien?

Comment les théâtres s'appellent-ils en italien?

?Comment les lieux s'appellent-ils en italien?

Comment les avocats s'appellent-ils en italien?

?Comment les êtres humains s'appellent-ils en italien?

Encore une fois, on constate que, par rapport aux exemples (a), les variantes (b) sont plus problématiques: les noms généraux objet, lieu et humain ont du mal à identifier des concepts qui puissent être dénommés. Ce constat est cohérent avec l'idée que ces concepts... n'existent pas, et que les contenus des noms sommitaux sont de toute autre nature par rapport aux concepts dénommés par guitare, théâtre ou avocat.

\subsubsection{Pseudo pronoms}

Nous soulignons ici un aspect particulier sous lequel le comportement des noms sommitaux se rapproche de celui des pronoms : le fonctionnement en tant que socle d'une qualité. Observons les exemples suivants :

C'est une personne insupportable.

Il est quelqu'un d'insupportable.

Les exemples (14) - mettant en jeu un nom sommital et un pronom - ne peuvent pas être analysés comme (15) car les coordinations (15) ne sont pas bien formées :

?C'est une personne et il est insupportable.

?Il est quelqu'un et il est insupportable.

Le dysfonctionnement de (15) suggère que ni personne, ni quelqu'un supportent le poids informationnel d'une position prédicative: ils n'arrivent pas à attribuer une 
information pertinente au sujet et ils remplissent plutôt la fonction d'offrir un socle à l'adjectif insupportable.

Cela n'est pas vrai pour un nom comme avocat:

C'est un avocat insupportable.

C'est un avocat et il est insupportable.

En somme, l'incompatibilité des noms sommitaux avec la classification fait en sorte qu'ils paraissent 'tirer' vers les pronoms.

\subsection{Conclusion intermédiaire}

Les phénomènes illustrés sous $\$ 2.2$ ont tous été discutés dans la littérature spécialisée. Nous renvoyons encore aux contributions de Mihatsch (2007), Gross (2009), Adler (2012), Fasciolo (2012), Mihatsch (2015a,b), Schnedecker (2015) Mihatsch (2017), Adler (2017) et Gerhard-Krait \& Vassiliadou (2017). En somme, le fait que les noms sommitaux soient récalcitrants à classifier a été très bien documenté.

Cependant, ce fait ne peut pas être valorisé qu'en reconnaissant, au préalable, que «classifier» est la fonction spécifique des noms (cf. §1). C'est seulement sur cette prémisse qu'on peut poser la question : "si la fonction des noms est de classifier et si les noms sommitaux ne classifient pas, quelle est leur fonction?»Cette question n'est pas explicitement posée par la littérature spécialisée, qui se contente de décrire le comportement des noms sommitaux par différence par rapport aux autres. A ce propos, des étiquettes telles que « hyper-classes » ou « classifieurs » sont révélatrices. Ne remettant pas en cause la fonction de classification, elles sont des véritables obstacles à chercher une fonction alternative pour les contenus des noms sommitaux. En insistant sur la généralité, elles envisagent la difficulté à classifier comme une conséquence de cette dernière, et non comme l'indice d'une autre fonction.

Mais il y a aussi une autre question. Revenons à l'argument « cognitif» (sous §2.1.2). Si la notion de prototype est un modèle du fonctionnement de nos concepts naturels et si les noms sommitaux s'avèrent incompatibles avec cette notion, alors, en toute logique, leurs contenus ne sont pas... des concepts. Si prise au sérieux, cette conclusion peut paraître déroutante car, si la signification d'un nom n'est pas un concept... qu'est-ce qu'elle est ? Est-il possible d'envisager l'existence de contenus qui ne relèvent pas du domaine de la « cognition »? Quelle serait leur nature ?

Notre conclusion intermédiaire se compose donc des interrogatifs suivants : $i$ ) Si le sens des noms sommitaux ne sert pas à classifier, à quoi sert-il ? ii) Si le sens des noms sommitaux n'est pas un concept, qu'est-ce qu'il est?

\section{Des hiérarchies aux restrictions de sélection}

La problématique des noms sommitaux est généralement abordée du point de vue des noms : comme une problématique concernant les " hiérarchies-être ». Or, si on contraste des expressions nominales telles qu'être vivants et insectes, on se limite à constater que les 
seconds sont inclus dans les premiers : la seule différence paraît concerner la généralité des notions impliquées, et non leur fonction.

C'est pourquoi nous proposons d'aborder la problématique des noms sommitaux non pas directement du côté des noms, mais bien des prédicats - ou relations - où ces noms peuvent entrer comme arguments. Cette perspective a un avantage sur la précédente. Si on contraste tuer un être vivant avec écraser un insecte, on peut examiner les différents types de conflits (au sens de Prandi 1998, 2017) qui découlent de la frustration des « restrictions de sélection ». Par là on peut remonter à la fonction spécifique des noms sommitaux et des prédicats qui les prennent comme arguments.

\subsection{Une reformulation du problème}

Considérons les couples d'exemples suivants :

Grand-père a écrasé une mouche.

Le boucher a abattu une vache.

Le jardinier a tondu le gazon.

Le bûcheron a abattu le chêne.

Les prédicats écraser, tondre et abattre (dans les emplois à chaque fois pertinents) prennent comme seconds arguments appropriés des entités appartenant aux ensembles des insectes, des têtes de bétail, des herbes et des arbres. Ces ensembles sont bien des classes sémantiquement homogènes et conceptuellement cohérentes au sens discuté sous $\S 2$. Dans les termes de Gross (2012), ce sont des «classes d'objets » identifiées par des emplois « appropriés » des prédicats en jeu. Puisque (dans ces emplois) les prédicats écraser, tondre et abattre circonscrivent des classes, nous disons qu'ils sont «fonctionnels à la classification ».

Considérons maintenant les exemples suivants :

Grand-père a tué une mouche.

Le boucher a tué une vache.

Le jardinier a coupé le gazon.

Le bûcheron a coupé l'arbre.

Les prédicats tuer et couper prennent comme seconds arguments tout sorte d'entité appartenant aux ensembles des êtres vivants et des objets concrets : tuer... une mouche, une vache, une bactérie, un lion, une plante, un otage, etc., couper... du gazon, un arbre, du papier, du tissu, un bloc de marbre, des cheveux, etc. Ces ensembles, c'est évident, n'identifient pas des véritables classes : être vivant ou objet concret sont bien des noms sommitaux incapables de classifier au sens du $\S 2$. Nous disons donc que les prédicats tuer et couper « ne sont pas fonctionnels à la classification ».

Notre problématique peut être reformulée comme il suit: s'il y a des prédicats qui ne sont pas fonctionnels à la classification - i.e. à créer des classes lexicales sémantiquement homogènes ou «classes d'objets »-quelle est leur fonction? 


\subsection{Conflits conceptuels}

Nous allons examiner les deux axes - horizontal et vertical - du lexique. Étant donné un prédicat du type verbe-c.o.d., nous examinons, d'abord, la relation entre le verbe et le complément (sous §3.2.1.) et, ensuite, la disponibilité d'un hyperonyme du verbe (sous §3.2.2.).

\subsubsection{Axe horizontal : correction vs. interprétation}

Commençons par observer un conflit au niveau des relations : écraser un insecte, abattre une tête de bétail, tondre de l'herbe et abattre un arbre:

*Grand-père a abattu une mouche.

* Le boucher a écrasé une vache.

*Le jardinier a abattu le gazon.

*Le bûcheron a tondu le chêne.

Les exemples (21) et (22) manifestent une anomalie qui peut être « corrigée ». Par là, nous entendons que le lexique offre un substitut approprié pour remplacer le verbe : ce substitut est le verbe correspondant dans les exemples (17) et (18) : abtre une mouche $\rightarrow$ écraser, écraser une vache $\rightarrow$ abattre, abatre le gazon $\rightarrow$ tondre, abattre.

Dans la mesure où les exemples (21) et (22) peuvent être corrigés, nous sommes confrontés à des fautes lexicales. Ces fautes ne consistent pas dans des procès conceptuellement incohérents ou absurdes car on comprend tout-à-fait la signification de (21) et (22). Ces fautes consistent simplement dans la violation des relations syntagmatiques (collocations ou solidarités) entre les verbes écraser, abattre et tondre d'une part et les classes des insectes, têtes de bétail, herbes et arbres de l'autre.

Passons maintenant à un conflit au niveau des relations tuer un être vivant et couper un objet concret :

* Grand-père a écrasé la lune.

*Le boucher a abattu la lune.

*Le jardinier a abattu la mélancolie.

*Le bûcheron a tondu la mélancolie.

Les exemples (23) et (24) manifestent une anomalie qui ne peut pas être corrigée au sens défini pour (21) et (22). En effet, cette fois, quel est le substitut approprié d'écraser, d'abattre ou de tondre ? Peut-être, voudrait-on répondre quelque chose comme détruire ou éliminer. Cependant, celles-ci ne sont pas des corrections, mais plutôt des « interprétations ». En (23) et (24), nous ne sommes plus confrontés à des fautes lexicales (qui brisent des collocations ou des solidarités), mais à des procès conceptuellement incohérents ${ }^{\mathrm{v}}$. 
Le point crucial est que les oppositions (17) vs. (21) et (18) vs. (22) - c'est-à-dire l'opposition entre emplois appropriés/correctes et inappropriés/fautifs des prédicats présupposent l'absence du conflit qui affecte (23) et (24), c'est-à-dire la cohérence conceptuelle. Par exemple, écraser un insecte est correcte, alors qu'écraser une tête de bétail ne l'est pas (et l'on dirait plutôt abattre une tête de bétail), parce qu'un insecte et une tête de bétail sont tous les deux des êtres vivants. Autrement dit, les prédicats écraser et abattre peuvent avoir des emplois où ils restreignent leur arguments aux insectes et aux têtes de bétail - en créant ainsi ces classes lexicales - car insectes et têtes de bétails sont des êtres vivants.

\subsubsection{Axe vertical : disponibilité d'un hyperonyme}

Revenons à la relation verbe-c.o.d. et observons maintenant les hyperonymes possibles du verbe.

A partir des exemples (21) et (22) - conceptuellement cohérents, mais fautifs - on peut passer aux exemples (19) et (20), cohérents et non fautifs. Nous les reproduisons ici :

*Grand-père a abattu une mouche $\rightarrow$ Grand-père a tué une mouche.

* Le boucher a écrasé une vache $\rightarrow$ Le boucher a tué une vache.

*Grand-père a abattu le gazon. $\rightarrow$ Le jardinier a coupé le gazon.

*Le jardinier a tondu le chêne. $\rightarrow$ Le bûcheron a coupé l'arbre.

Comme on le voit, en passant aux hyperonymes tuer et couper, on dissout la faute qui affecte (21) et (22). C'est la preuve du fait que l'opposition «approprié vs. inapproprié »se fondait précisément sur les idées d'être vivant et d'objet concret impliquées par tuer et couper. Tuer un insecte et tuer une tête de bétail, par exemple, sont cohérents parce que tuer un être vivant est cohérent. Ce fait fonde la possibilité d'avoir des prédicats spécifiques pour exprimer les premiers et, par conséquent, la possibilité de les employer d'une façon incorrecte.

Une preuve a contrario est offerte par l'impossibilité de passer à un hyperonyme dans le cas de (23) et (24):

*Grand-père a écrasé la lune $\rightarrow$ *Grand-père a tué la lune.

*Le boucher a abattu la lune $\rightarrow *$ Le boucher a tué la lune.

*Le jardinier a abattu la mélancolie $\rightarrow$ *Le jardinier a coupé la mélancolie.

*Le bûcheron a tondu la mélancolie $\rightarrow$ *Le bûcheron a coupé la mélancolie.

En effet, cette fois, l'incohérence reste: aucun hyperonyme n'est disponible. Le conflit en (23) et (24) - à la différence de celui en (21) et (22) - ne repose donc pas sur une base cohérente : tout fondement est brisé. Par conséquent, a fortiori, il ne peut pas y avoir un prédicat approprié pour dire *tuer les étoiles plutôt que *tuer les satellites ou *couper les sentiments plutôt que *couper les émotions. 


\subsection{Conclusion générale}

Nous sommes maintenant en mesure de répondre à la question soulevée sous \$3.1. Rappelons les termes de cette question. D'un côté, il y a des prédicats qui sont fonctionnels à la classification: ils circonscrivent, dans des positions argumentales données, des ensembles d'entités qui identifient des véritables classes d'objets ou concepts. Ces classes correspondent aux contenus des noms communs prototypiques. De l'autre côté, il y a des prédicats qui ne sont pas fonctionnels à la classification : ils circonscrivent, dans des positions argumentales données, des ensembles d'entités qui n'identifient pas des véritables classes d'objets ou concepts. Ces ensembles correspondent aux contenus des noms sommitaux.

Or, les prédicats qui ne sont pas fonctionnels à la classification remplissent la fonction de fixer les conditions de cohérence que les autres prédicats doivent respecter pour constituer des véritables classes d'objets. Un prédicat comme tuer, en délimitant les êtres vivants en position de c.o.d., fixe les limites logiques à l'intérieur desquels le lexique peut tracer les classes des insectes et des têtes de bétail à travers certains emplois appropriés d'écraser ou d'abattre. Insectes et têtes de bétail - délimitées par écraser et abattre à l'intérieur des êtres vivants - sont bien des outils classificatoires forgés par le lexique. Cependant, l'ensemble des êtres vivants - reproduit par tuer - n'est pas lui-même un outil de classification, mais bien un " champ de cohérence » où le lexique est libre de construire ses outils de classification à travers des matrices de prédicats appropriés.

Selon nous, les noms sommitaux sont récalcitrants à classifier parce qu'ils sont la manifestation lexicale de ces champs de cohérence. La nature de leur contenu n'est pas «cognitive », mais «ontologique »: ils sont les indices d'une ontologie naturelle partagée - sur la base de laquelle chaque lexique peut se différencier des autres, d'une façon arbitraire, par rapport à la configuration de ses classes d'objets.

Une façon pour distinguer ces deux niveaux consiste à opposer «classes » $v s$. «catégories », les noms sommitaux se rapprochant de ces dernières. Une catégorie au sens kantien (par exemple, la causalité) n'identifie pas une classe de phénomènes, mais bien les relations possibles entre les phénomènes. De même - mutatis mutandis - des notions telles qu'être vivant, objet concret, lieu, etc. n'identifient pas des classes d'objets, mais bien l'espace logique des classes d'objets possibles.

Une autre façon pour distinguer les niveaux susmentionnés consiste à utiliser la distinction de Searle (1979) entre Network (nos concepts, nos croyances encyclopédiques) et Background: " a set of non-representational mental capacities that enable all representing to take place ». Les classes d'objets appartiennent au domaine du Network, alors que les notions sommitales appartiennent au domaine du Background. Cette perspective a l'avantage de mettre en évidence que - loin d'être les sommets du lexique ces notions en sont en réalité les fondements. C'est pourquoi nous proposons de les appeler « noms du fond » (ground nouns, an.).

\section{Perspectives}

\subsection{Prédicats universels}


Sous \$3.2.1, nous avons affirmé que dans un exemple tel que le boucher a tué la mélancolie, nous ne sommes pas confrontés à une « faute lexicale ». Cela revient à affirmer que la sélection du domaine des êtres vivants effectuée par tuer sur le c.o.d., ne découle pas du lexique, mais d'une ontologie naturelle que ce prédicat se limite à reproduire. Ce fait a deux conséquences.

La première conséquence concerne l'application de la notion de « restrictions de sélection». Cette notion indique des restrictions combinatoires de nature lexicale ou syntaxique. En ce sens, elle peut être appliquée correctement à la sélection de la classe des tètes de bétail de la part du prédicat abattre - où il y a la possibilité d'une faute lexicale mais non à tuer des êtres vivants. Le choix de l'étiquette « restrictions de sélection » pour le titre du $\S 3$ était donc motivée seulement par des raisons de praticité.

La seconde conséquence est que si un prédicat comme tuer des êtres vivants reproduit une relation ontologique préalable au lexique, il est raisonnable de s'attendre à ce qu'il soit universel. Cela suggère d'enquêter le caractère universel des notions du fond en se concentrant, encore une fois, non pas directement sur la présence de certains noms, mais sur la présence de certains prédicats. Peu importe si une langue ait ou pas un mot pour dire êtres vivants. Ce qui compte c'est qu'elle ait un prédicat comme tuer prenant les êtres vivants comme arguments cohérents (et des prédicats appropriés comme écraser qui découpent des classes à l'intérieur d'un tel champ).

\subsection{Champs de cohérence}

Sous $\$ 3.3$, pour décrire la fonction des noms du fond, nous avons utilisé la métaphore du « champ de cohérence ». Il y a un constat qui paraît justifier cette métaphore.

Humain, objet concret, être vivant, être animé, etc. ne s'opposent pas entre eux, mais chacun fonctionne comme un pôle logique. Des prédicats tels que couper, mourir, souffrir ou s'indigner, par exemple, génèrent un champ cohérent (des concrets, des vivants, des animés et des humains) opposé à tout le reste qui s'avère incohérent (les non-concrets, les non-vivants, les non-animés et les non-humain). De ce point d vue, ce n'est pas par hasard que les restrictions de sélection de la grammaire générative des origines étaient formulées en termes de +- humain, +- animé, +- concret, etc.

En revanche, les classes d'objets - définies par des prédicats appropriées - tendent à s'opposer entre elles (dans un champ délimité par les noms du fond). Les transports publiques s'opposent aux transports privés, les herbes s'opposent aux arbres, les têtes de bétail s'opposent à d'autres animaux domestiques ou aux animaux sauvages, les meubles s'opposent aux biens immeubles et ainsi de suite.

\subsection{Dimensions du sens}

Sous §2.2.2, nous avons rappelé que les noms du fond supportent mal le poids informationnel d'une position prédicative. Contrastons, par exemple, désert et lieu :

Le Sahara c'est un désert.

?Un désert c'est un lieu. 
Dans la mesure où il est pertinent d'affirmer (23a), il est également pertinent d'identifier une hiérarchie de type "hyponyme < hyperonyme » entre Sahara et désert. Vice-versa, dans la mesure où affirmer (23b) n'est pas informatif, identifier une hiérarchie de ce type entre désert et lieu perd d'intérêt. Certes, en principe, rien n'empêche de considérer un désert comme un sous type de lieu. Cependant, lexicalement, lieu ne paraît pas fonctionner comme un bon hyperonyme de désert (foret, vallée, plaine, etc.). Autrement dit, lieu n'est pas le sommet d'une hiérarchie-être comme « Sahara < désert ... $<* l i e u$ ». Encore une fois, le fait que ces noms paraissent lexicalement déconnectés des autres noms a été remarqué ; les conséquences de ce fait, cependant, n’ont pas été toutes tirées.

D'une part, la remarque précédente confirme l'inadéquation de la dénomination même « noms sommitaux » car tout sommet présuppose une continuité avec sa base. D'autre part, elle soulève à nouveau notre question centrale : si lieu n'est pas le dernier anneau d'une chaîne hiérarchique, quelle est sa fonction?

Observons les exemples suivants :

Paul a déménagé son armoire dans sa maison de montagne.

Paul a rangé ses chemises dans l'armoire.

Un armoire est un meuble: l'emploi du prédicat déménager en (24a) est en effet approprié à cette classes d'objets. L'énoncé (24b), quant à lui, présente un exemple basique du phénomène étudié sous les noms de «coercition » (exploitation, cf. Pustejovsky\&Jezek 2008), «modulation» (Cruse 1986) et «métonymie intégrée» (Kleiber 1999). Très synthétiquement, en (24b), le prédicat ranger touche la facette locative d'armoire ${ }^{\mathrm{vi}}$. Des remarques pareilles sont valables pour moyen de transport public et objet concret dans les exemples suivants :

Paul a pris le train.

Ce train pèse 230 tonnes.

Or, si lieu et objet concret ne fonctionnent pas comme véritables sommets des hiérarchies " armoire $<$ meuble $<\ldots$. ou «train $<$ moyen de transport $<\ldots$ » à quoi servent-ils ? Le phénomène de la coercition suggère qu'ils ne font pas partie du sens des mots, mais ils offrent des dimensions - des catégories - par rapport auxquelles le sens des mots se plie ou bouge. De ce point de vue, ce n'est pas par hasard que les Qualia sont des notions fondamentales telles que matière, agent, fonction....

\subsection{Double statut des humains}

Sous §2.1.2, nous avons souligné la difficulté des noms du fond à fonctionner comme des concepts capables de classifier des occurrences plus ou moins prototypiques. A ce propos les humains sont un cas particulier. Certes, on ne le fera peut-être pas dans la vie de tous les jours, mais il paraît quand-même sensé d'affirmer : "un martien est comme un humain, mais avec des antennes vertes, etc. ». En tout cas, il parait beaucoup plus sensé que pour 
lieu ou objet concret. Par ailleurs, à la différence de ce qui se passe avec ces dernières notions, il est tout à fait possible de dessiner le prototype d'un être humain. Faut-il conclure qu'après tout les êtres humains ne sont pas une catégorie ontologique ? Faut-il en conclure qu'ils ont un statut ambigu? Comme on le voit, les humains méritent bien l'attention particulière qui leur a été consacrée (cf. Schnedecker 2015).

Nous croyons que les critères employés sous $\S 3$ peuvent aider à cerner ce problème. Commençons par observer que l'incohérence d'un exemple comme *La mer est indignée montre clairement que les humains peuvent fonctionner en tant que catégorie ontologique. Le problème vient de la comparaison entre (26) et (17b) :

* Le boucher a assassiné une vache.

Le boucher a abattu une vache.

Le boucher a tué une vache.

Il n'est pas difficile de se rendre compte que, dans les termes introduits sous $\S 3.2$, l'exemple (26) est un emploi inapproprié - et non incohérent - du prédicat assassiner. Il s'en suit que la classe des êtres humains, circonscrite par ce prédicat, est en effet une classe d'objets qui s'oppose aux têtes de bétails à l'intérieur du champ des êtres vivants ${ }^{\mathrm{vii}}$.

Les êtres humains manifestent donc un double statut : ils fonctionnent comme catégorie ontologique par rapport à un prédicat tel qu'être indigné et ils fonctionnent comme classe d'objets - découpée à l'intérieur du champ des êtres vivants - par rapport à un prédicat tel qu'assassiner. Remarquons qu'il y a d'autres cas où les humains fonctionnent comme une classe lexicale circonscrite par des prédicats appropriés. En Français, il y l'opposition cadavre (humains) vs. carcasse (animaux) à l'intérieur de la catégorie ontologique des êtres animés. En Allemand, toujours dans le cadre de la catégorie ontologique des êtres animés, il y a la distinction entre Essen (manger, humains) et Fressen (manger, animaux). En Anglais, to murder est approprié pour tuer des humains, alors que to assassinate est approprié pour tuer des hommes politiques.

Sur la base du constat de ce double statut, on pourrait faire l'hypothèse que les humains identifient justement le point de jonction entre classes lexicales et catégories ontologiques. Cette position de carrefour constituerait l'une de leurs particularités.

Cet article voudrait être un hommage et un remerciement à G. Kleiber, explorateur de sommets lexicaux. Le soleil fait de l'ombre à ceux qui ne regardent que leurs pieds

\section{Références bibliographiques}

Adler, S. (2012). Trois questions relatives aux noms généraux factuels attitudinaux. Scolia, 26, 11-38.

Adler, S. (2017). Les noms généraux - «shell nouns »- participent-ils à une lecture taxinomique de type Hiérarchie-être ? Syntaxe et sémantique 18, 1, 45-63.

Aikhenvald, A. Y. (2000). Classifiers. A Typology of Noun Categorization Devices. Oxford : OUP. 
Cruse, D. A. (1986). Lexical semantics. Cambridge : CUP.

Fasciolo, M. (2012). Y-a-t-il un continuum entre noms et pronoms ?. Scolia, 26, 61-80.

Gerhard-Krait, F. \& Vassiliadou, H. (2017). Lectures taxinomique, approximative et floue : quelques pistes supplémentaires, Syntaxe et sémantique 18, 1, 11-18.

Greenberg J. H. (1972). Numeral Classifiers and Substantival Number : Problems in the Genesis of a Linguistic Type, Working Papers on Language Universals, 9, 1-39.

Gross G. (2008). Les classes d'objets. Lalies, 28, 111-165.

Gross G. (2009). Sur le statut des substantifs humains. In : Leeman D. (éd.). Des topoï à la théorie des stéréotypes en passant par la polyphonie et l'argumentation. Hommages à Jean-Claude Anscombre. Chambery : Université de Savoie, 27-41.

Gross G. (2012). Manuel de linguistique. Ville-Neuve d'Ascq, Presses Universitaires du Septentrion.

Halliday M. A. K. \& R. Hasan (1960), Cohesion in English, London : Longman.

Harris Z. S. (1976). Notes du cours de syntaxe (traduit par M. Gross), Paris : Editions du Seuil.

Huyghe, R. (2009). Les noms généraux d'espace en francais - enquete linguistique sur la notion de lieu. Paris : De Boeck - Duculot.

IVANIČ R. (1991), Nouns in search of a context : A study of nouns with both open- and clodes-system characteristics. "International Review of Applied Linguistics in Language Teaching », 2, 93-114.

Kleiber, G. (1987). Dénomination et relations dénominatives, Langages, 73, 77-94.

Kleiber G. (1994). Lexique et cognition: Y a-t-il des termes de base ?, Scolia 1, 7-40.

Kleiber, G. (1999). Problèmes de sémantique. La polysémie en questions, Ville-Neuve d'Ascq, Presses Universitaires du Septentrion.

Kleiber, G. et Riegel, M., 1978, Les grammaires floues, in Martin, R. (éd.), La notion de recevabilité en linguistique, Paris, Klincksieck, 67-124.

Lakoff, G., (1972), Hedges : a study in meaning criteria and the logic of fuzzy concepts, Chicago Linguistic society, 8, 183-228.

Lyons J. (1977, 1996), Semantics $1 \& 2$, Cambridge : Cambridge University Press.

Mahlberg M. (2005). English general nouns ; a corpus theoretical approach. Amsterdam : John Benjamins.

Mihatsch, W. (2007). Taxonomic and meronimic superordinates with nominal coding. In : Schalley, A. C. \& Zaefferer D. (éd.) Ontolinguistics. How Ontological Status Shapes the Linguistic Coding of Concepts. Berlin- NewYork : Mouton de Gruyter.

Mihatsch, W. (2015a). La sémantique des noms généraux 'être humain' français et allemands. In : W. Mihatsch \& C. Schnedecker C. (éds), Les noms d'humains : une catégorie à part? Stuttgart, Steiner, 55-83.

Mihatsch, W. (2015b). La position taxinomique et les réseaux meronyiques des noms généraux des noms généraux 'être humain' français et allemands. In : W. Mihatsch \& C. Schnedecker C. (éds), Les noms d'humains : une catégorie à part? Stuttgart, Steiner, 85-113.

Mihatsch, W. (2017). Les noms d'humains généraux aux limites de la grammaticalisation, Syntaxe et sémantique 18, 1, 67-99.

Mihatsch W. \& Schnedecker C. (ed.) (2015), Les noms d'humains : une catégorie à part? Franz Steiner Verlag.

Prandi M. (1998). Contraintes conceptuelles sur la distribution: réflexions sur la notion de classe d'objets, Langages, 131, 34-44.

Prandi M. (2017). Conceptual Conflicts in Metaphors and Figurative Language. New York - London : Routledge.

Pustejovsky, J (1995 ). The Generative Lexicon, Cambridge-Massachussets: MIT. 
Pustejovsky, J. \& Jezek E. (2008), Semantic Coercion in Language: Beyond Distributional Analysis, Rivista di Linguistica, 20.1, 181-214.

Quine, W. O. (1953, 2003). Du point de vue logique. Paris: Vrin.

Rosch et alii (1976), Basic Objects in Natural Categories, Cognitive Psychology, 8, 382436.

Schnedecker C. (2015). Les (noms d') humains sont-ils à part ? Des intérêts et perspectives linguistiques d'une sous-catégorie nominale encore marginale, in W. Mihatsch \& $\mathrm{C}$. Schnedecker C. (éds), Les noms d'humains : une catégorie à part ? Stuttgart, Steiner, 4-43.

Searle, J. R. (1979). Expression and Meaning: Studies in the theory of Speech Acts. Cambridge: CUP.

\begin{abstract}
i Par rapport à la classification, les noms prototypiques s'avèrent les noms communs et non les noms propres. Le fait qu'une partie du discours ait une fonction qui n'est pas partagée par tous ses membres n'est pas surprenant et ne remet pas en cause son intégrité. La fonction prototypique des verbes, par exemple, est de relier des arguments (i.e. avoir une valence), mais cela n'empêche ni que certains verbes fonctionnent comme copules ou supports, ni qu'autres parties du discours puissent avoir une valence.
\end{abstract}

ii De ce point de vue, ce sont les groupes nominaux qui sont définis par rapport à la distribution des pronoms, et non vice-versa. C'est pourquoi Quine (1953) propose d'appeler les groupes nominaux « pro-pronoms ».

iii

Dans l'emploi ici en jeu, il serait donc fourvoyant d'affirmer une poule est une sorte d'oiseau parce qu'une poule.... est un oiseau! Une telle affirmation, en revanche, est tout à fait correcte dans la lecture inclusive (ici non pertinente) : une poule est l'un des types d'oiseau. Remarquons que la version négative sélectionne la lecture inclusive : une mandoline est une sorte de guitare (approximation ou inclusion) vs. une mandoline n'est pas une sorte de guitare (inclusion). En effet, d'un point de vue conceptuel, l'approximation est déjà une opération négative. Il paraît alors raisonnable qu'une forme linguistiquement négative n'ait pas beaucoup de sens. Le débat sur l'approximation, le flou, l'inclusion etc. est extrêmement riche, et dans cette contribution nous ne pouvons que le côtoyer. Nous renvoyons à l'introduction de Gerhard-Krait, F. \& Vassiliadou, H. (2017).

iv La nuance s'impose car, par rapport à ce type de test, les humains manifestent un comportement moins net. Nous y reviendrons sous $\S 4.4$.

v Pour les notions de cohérence, incohérence conceptuelle et conflit, nous renvoyons à Prandi (2017).

vi On pourrait imaginer que la catégorie de lieu appliquée à un meuble produit un endroit. Quoi qu'il en soit, pour la distinction fine entre lieu, endroit, etc., nous renvoyons à Huyghe (2009)

vii Abattre a également un emploi approprié aux humains (Ils ont abattu le condamné), mais cela n'est pas pertinent pour notre discours qui concerne ici assassiner. 